\title{
Identification and Characterization of Copy Number-Associated Driver Genes in Esophageal Squamous Cell Carcinoma
}

\author{
Kexin Jiang, Yijiang He, and Xu Zhang \\ Radiation Oncology Department of Gastrointestinal Cancer and Lymphoma, Cancer Hospital of China Medical University, \\ Liaoning Cancer Hospital and Institute, China \\ Correspondence should be addressed to Xu Zhang; posi29937@163.com
}

Received 15 June 2020; Accepted 17 July 2020; Published 24 August 2020

Guest Editor: Tao Huang

Copyright (c) 2020 Kexin Jiang et al. This is an open access article distributed under the Creative Commons Attribution License, which permits unrestricted use, distribution, and reproduction in any medium, provided the original work is properly cited.

\begin{abstract}
Background. Esophageal squamous cell carcinoma (ESCC) is a leading malignancy with both high incidence and mortality worldwide. However, the molecular mechanisms of the poor prognosis in ESCC are still unclear. Methods. We conducted differential expression analysis between ESCC and normal tissues and between ESCC samples with and without CNAs in a given gene. Overrepresentation enrichment and gene set enrichment analyses were used to identify the oncogenic pathways and abnormal transcription factors (TFs). The survival analysis was employed to identify the genes associated with overall survival. Results. In this study, we aimed to identify and interpret the driver genes triggered by the copy number alterations (CNAs), including CCND1, TEAD4, EIF4EBP1, EGFR, FGFR3, and FZD6. Furthermore, we identified oncogenic pathways, including RTK-RAS, WNT, PI3K, Hippo, and cell cycle, and key TFs including TEAD4, a transcription factor in the Hippo signaling pathway, and LEF1 in the WNT signaling pathway. Furthermore, we observed that upregulations of FGFR3 and EIF4EBP1 were significantly associated with shorter overall survival in ESCC. Conclusion. In conclusion, the driver genes triggered by CNAs not only exhibited critical functionality but also were clinically relevant in ESCC, which greatly improved our understanding of the molecular mechanisms in ESCC.
\end{abstract}

\section{Introduction}

Esophageal cancer is the seventh most prevalent malignancy worldwide and ranked sixth in mortality [1] (6.6\% of the total cancer deaths). Esophageal cancer mainly consists of two types: esophageal adenocarcinoma (EAC) and esophageal squamous cell carcinoma (ESCC), with ESCC accounting for the majority of esophageal malignancies [2]. ESCC is considered to be associated with environmental risk factors, such as chronic smoking, hot tea drinking, and red meat and alcohol consumption, and previously proposed precursor lesions of ESCC including esophagitis, atrophy, and dysplasia $[3,4]$. Though advanced screening techniques have greatly helped the diagnosis of ESCC, it is worth noticing that even for patients with an early-staged ESCC, recurrence or distant metastases shortly after surgery can be observed, which often leads to poor prognosis [2]. In order to develop individualized and targeted therapies for ESCC, molecular mechanism behind ESCC carcinogenesis and metastasis needs to be further clarified.

With next-generation sequencing (NGS) technology, investigation of the genetic background has unveiled the involvement point mutations, insertions, deletions, and copy number alterations/variations (CNA/CNV) in ESCC tumorigenesis. So far, a previous study has demonstrated the interaction between ADH1B and/or ALDH2 risk alleles and environmental risk factor through SNP genotyping in ESCC [5], and another study has identified 26 significantly mutated genes in ESCC, where TENM3 mutations and the TP53 hotspot mutation p.R213* were stated to be related to poor prognosis [6]. Notably, nonsynonymous mutation and copy number loss/gain in TP53, KMT2D, NOTCH1, PIK3CA, and FAT1 in ESCC are frequently reported and discussed, as they played important roles in NOTCH signaling, the RTK-MAPK-PI3K pathway, and cell cycle regulation, all of which were closely related to tumor development and differ- 
entiation [7-9]. Moreover, a recent study has demonstrated the association between NTRK1 gene amplification and the efficacy of larotrectinib in a metastatic ESCC patient, which hinted that exploring novel $\mathrm{CNA} / \mathrm{CNV}$ and related driver mutations would provide insights for making therapeutic decisions [10]. In the present study, we conducted a systematic data analysis to identify the driver genes and characterize their functionalities in ESCC, which might provide some evidences for the related research studies.

\section{Materials and Methods}

2.1. Data Acquisition. From the UCSC Xena database (http:// xena.ucsc.edu/), we downloaded the gene expression data and copy number alterations (CNAs) of ESCC patients, which were collected by the Cancer Genome Atlas (TCGA, https://portal.gdc.cancer.gov) project $[11,12]$. The expression levels were normalized at FPKM (fragments per kilobase per million) and log2-transformed. The copy number status of gains or losses and the wild type were determined by their $\log 2$ (copy number/2). The gene expression dataset for the independent validation of differential expression was collected from Gene Expression Omnibus (GEO) with accession number GSE45670 [13].

2.2. Differential Expression Analysis. The 81 esophageal squamous cell carcinoma (ESCC) and 11 adjacent normal tissues with detailed clinical information from TCGA were used for the differential expression analysis. We applied the Wilcoxon rank-sum test and calculated the fold change to determine if the differences observed were statistically significant, and genes with an adjusted $P$ value less than 0.05 and a fold change higher than 2 were considered the differentially expressed genes (DEGs).

2.3. Copy Number Alteration-Related Driver Genes. First, we used gene entries from the GENCODE (https://www .gencodegenes.org/) v22 database to annotate the segmented CNAs that we obtained [14]. Utilizing these segmented CNAs, we estimated and assigned values for their corresponding genes, which were calculated as $\log 2$ (copy number/2). The expressions of these genes were then taken into consideration. For each of these genes, we measured its differential expression levels between the samples with and without CNAs, and the threshold was set to a $P$ value $<0.0001$ to identify the driver genes triggered by the corresponding CNAs.

2.4. Overrepresentation Enrichment Analysis (ORA). After identifying these differentially expressed genes between ESCC and normal tissues, we performed an overrepresentation enrichment analysis on the obtained gene set. Enricher function in R clusterProfiler package was used to carry out ORA [15], and an adjusted $P$ value lower than 0.05 was considered statistically significant [16]. The genes in each oncogenic pathway were obtained from $\mathrm{R}$ package maftools.

2.5. Cox Proportional Hazard Regression Analysis. We used package survival in $\mathrm{R}$ to conduct survival analysis. To identify driver genes that could significantly affect overall survival, we performed univariable Cox regression and plotted Kaplan-
Meier curves to demonstrate varied survival probabilities among different groups using $\mathrm{R}$ package survminer.

\section{Results}

3.1. Identification of Differentially Expressed Genes between Esophageal Squamous Cell Carcinoma and Adjacent Normal Tissues. To detect the differentially expressed genes (DEGs) between 81 esophageal squamous cell carcinoma (ESCC) and 11 adjacent normal tissues from the Cancer Genome Atlas (TCGA), we compared the gene expression profiles and identified 2,160 upregulated and 3,057 downregulated genes (Figure 1(a)). The principal component analysis (PCA) of these genes revealed that the ESCC samples could be clearly distinguished from the adjacent normal tissues (Figure 1(b)). Results from functional enrichment analysis on those dysregulated genes hinted that activities of cell cycle-related pathways and metabolism-related pathways were significantly altered in ESCC (Figure 1(c)), respectively. These results suggested that the DEGs could not only differentiate the ESCC from normal tissues but also reveal cancerrelated pathways.

3.2. Identification of CNA-Triggered Driver Genes. To further detect driver genes triggered by the copy number alterations (CNAs), we examined whether the expression levels of DEGs were triggered by the CNAs. In total, we identified six driver genes including CCND1, TEAD4, EIF4EBP1, EGFR, FGFR3, and FZD6, which were highly upregulated in the samples with gains of the corresponding genes (Figure 2(a), Wilcoxon rank-sum test, $P<0.05)$. Particularly, FGFR3 and EGFR were involved in the RTK-RAS pathway, and the remaining driver genes including FZD6, EIF4EBP1, TEAD4, and CCND1 participated in the WNT, PI3K, Hippo, and cell cycle pathways (Figure 2(b)). Furthermore, to validate the dysregulation of these genes in ESCC, we also collected an independent gene expression dataset to investigate their differential expression levels between ESCC and adjacent normal tissues. Specifically, all these genes were highly upregulated in ESCC as compared to the normal tissues $(P<0.05$, Figure S1). These results indicated that the integrative analysis of the CNAs and gene expression data could identify the candidate driver genes and oncogenic pathways involved in ESCC.

3.3. The Consequences of CNA-Related Driver Genes on the Downstream Pathways. To further investigate the consequences of the CNA-related driver genes on the downstream pathways, we conducted overrepresentation enrichment analysis (ORA) to identify the oncogenic pathways that the DEGs were enriched in. Notably, WNT, NOTCH, and cell cycle were significantly upregulated in the ESCC samples (Figure 3(a)). The ligands, including WNT7A, WNT7B, WNT3A, WNT5A, and WNT2; the receptors, including FZD2, FZD6, and FZD10; and the transcription factor, LEF1, in the WNT signaling pathway, were significantly upregulated in ESCC (Figure 3(b)). Similarly, the components in the NOTCH signaling pathway and cell cycle were also significantly elevated in ESCC compared with the 

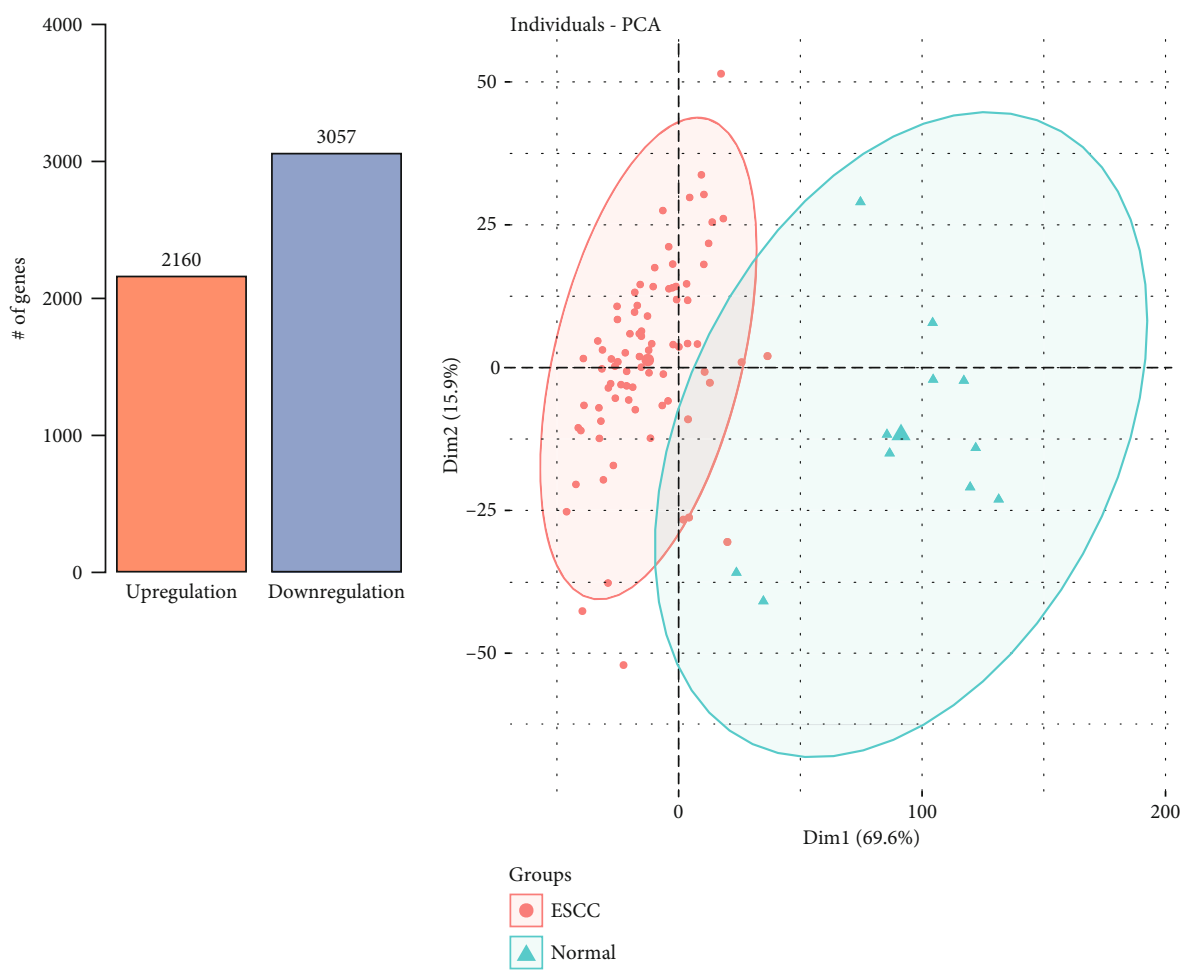

(a)

(b)

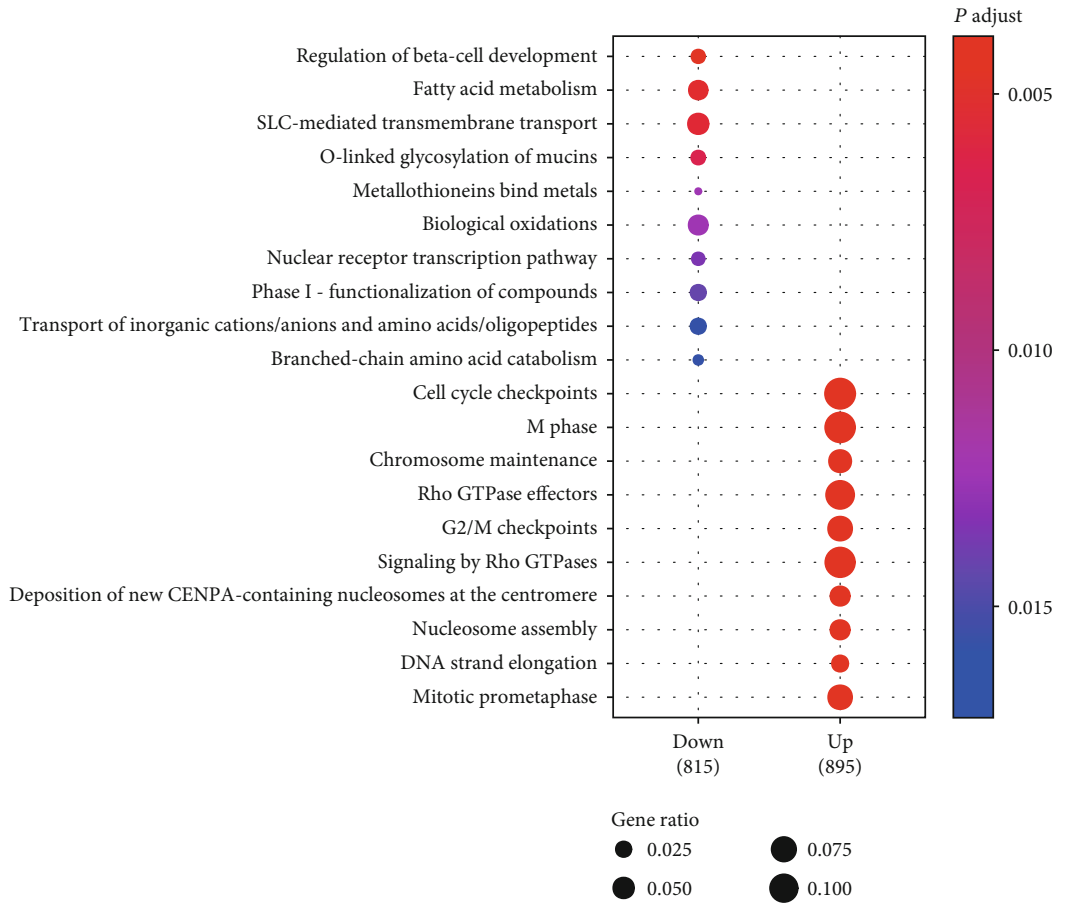

(c)

FIGURE 1: The differentially expressed genes and dysregulated pathways in ESCC. (a) The number of upregulated and downregulated genes in ESCC, which are represented by the orange and blue bars, respectively. (b) The ESCC and adjacent normal samples are represented by the nodes colored by red and green, respectively. The $x$ - and $y$-axes represent the top two principal components by principal component analysis (PCA). (c) The pathways enriched by the upregulated and downregulated genes. The node size and color represent the number of genes and statistical significance. 

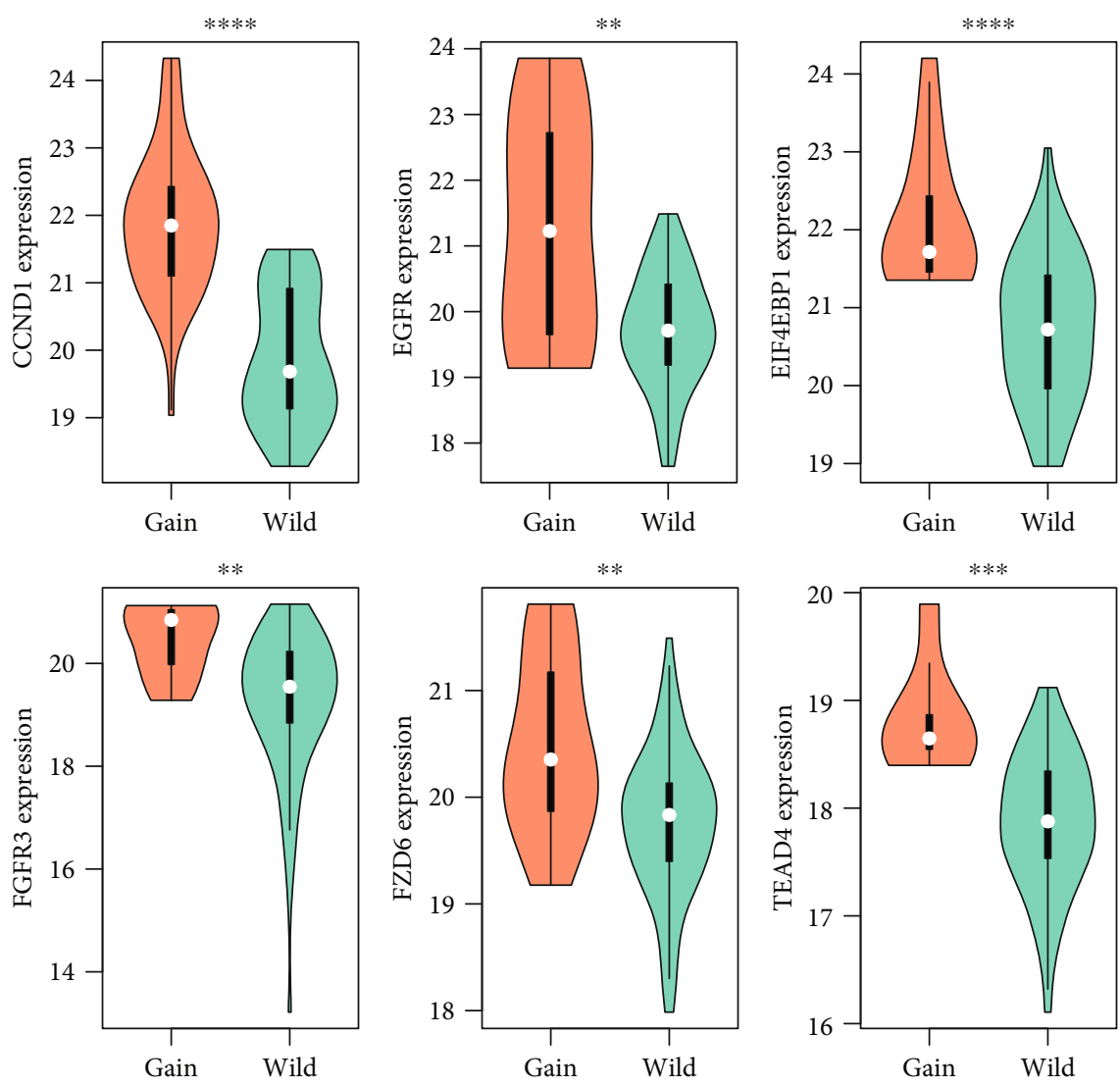

(a)
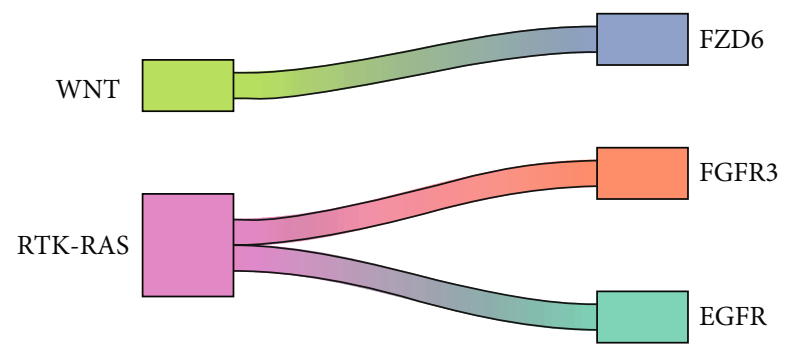

PI3K

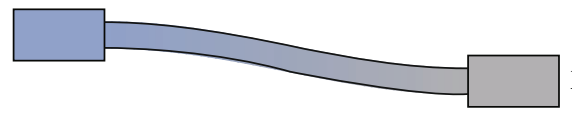

EIF4EBP1
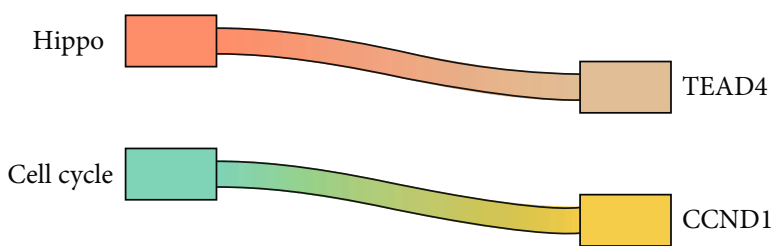

(b)

FIgURE 2: The driver genes and their regulating oncogenic pathways. (a) The expression levels of the CNA-related driver genes between the samples with and without CNAs. (b) The regulating oncogenic pathways that the driver genes were involved in. The symbols of $*, * *, * * *$, and $* * * *$ indicate the $P$ values less than $0.05,0.01,0.001$, and 0.0001 , respectively.

adjacent normal tissues (Figure 3(b)). Collectively, the abnormal upregulation of driver genes including FZD6 and CCND1 might result in activation of the WNT and cell cycle pathways.
3.4. The Key Transcription Factors of Driver Gene-Mediated Oncogenic Pathways. To further interpret and verify the functionalities of the CNA-related driver genes, we investigated whether the transcriptional regulatory activities of the 


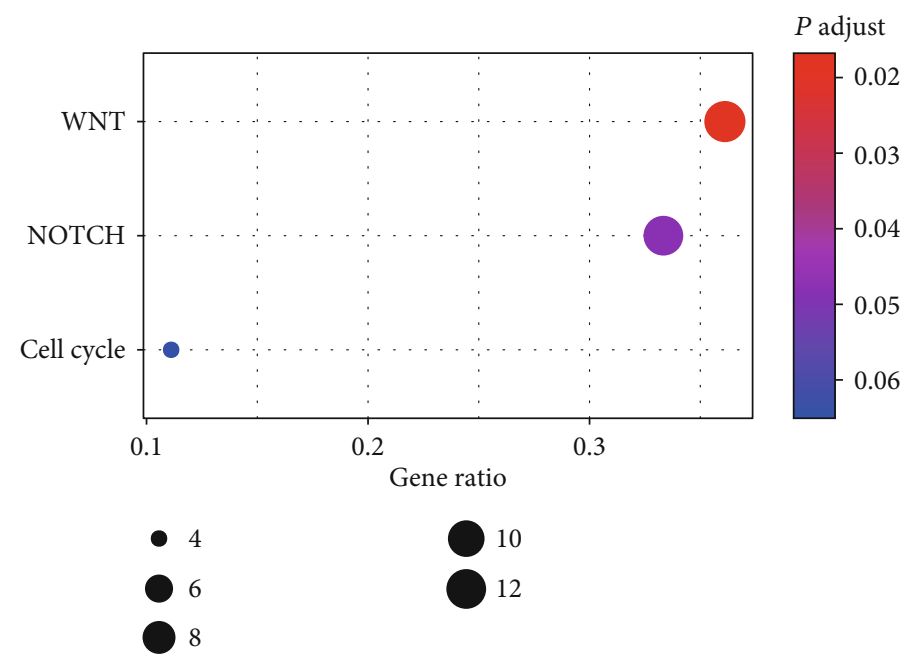

(a)
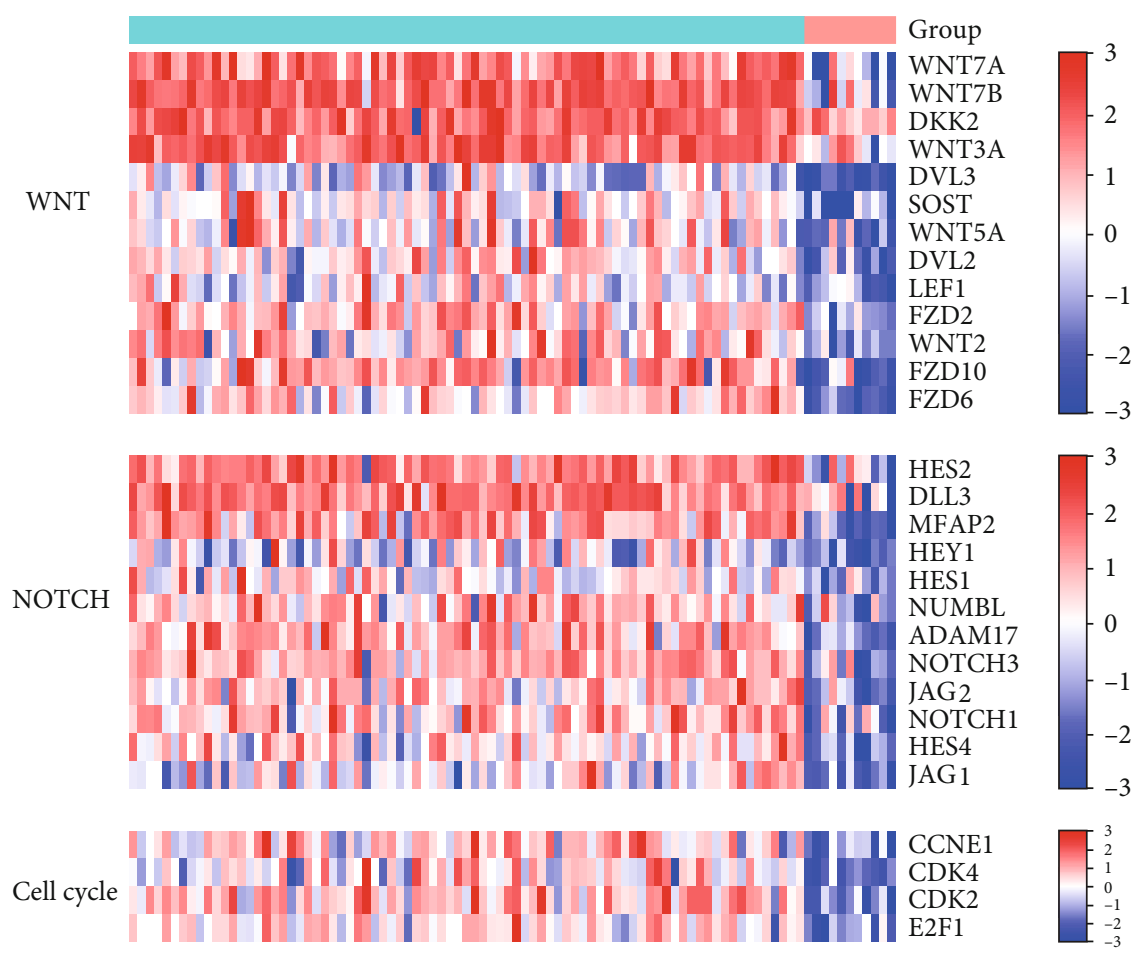

Group
ESCC
Normal FIGURE 3: The consequences of the driver genes on the oncogenic pathways. (a) The oncogenic pathways enriched by the upregulated and
downregulated genes. The node size and color represent the number of genes and statistical significance. (b) The dysregulated genes in the oncogenic pathways enriched by the differentially expressed genes.

transcription factors (TFs) were also activated in the oncogenic pathways. Among the TFs in the oncogenic pathways, the expression levels of LEF1 and TEAD4 were found to be significantly altered in ESCC samples and they participated in the abnormally activated oncogenic pathways such as WNT and Hippo signaling. Consistently, from the gene set enrichment analysis (GSEA), we observed that the target genes of LEF1 and TEAD4 were also significantly enriched by the upregulated genes in ESCC samples (Figures 4(a) and 4(b)). The abnormal activities of the downstream target genes of LEF1 and TEAD4 further suggested that the WNT and Hippo signaling pathways were abnormally activated by the driver genes FZD6 and TEAD4, respectively. 


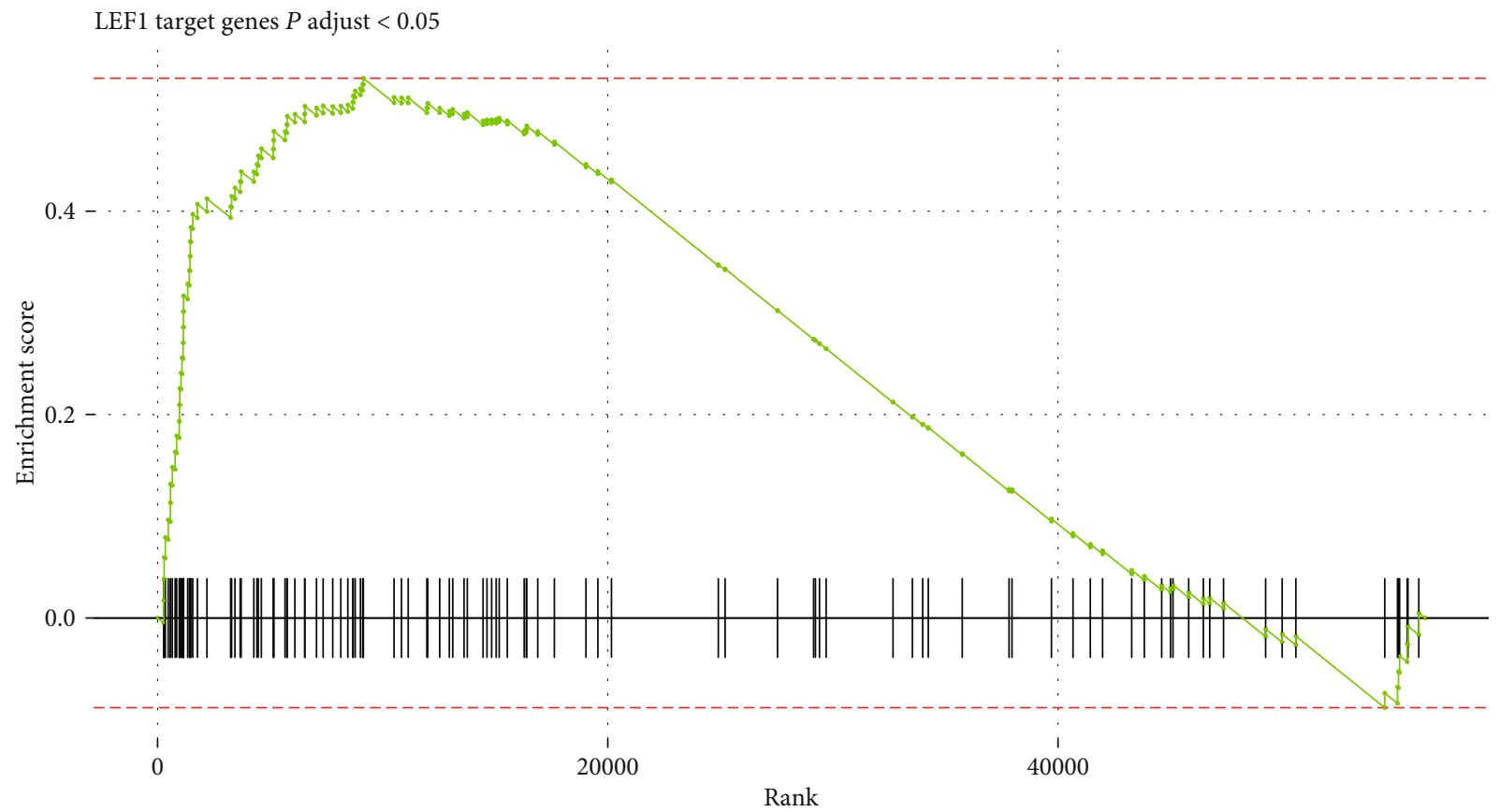

(a)

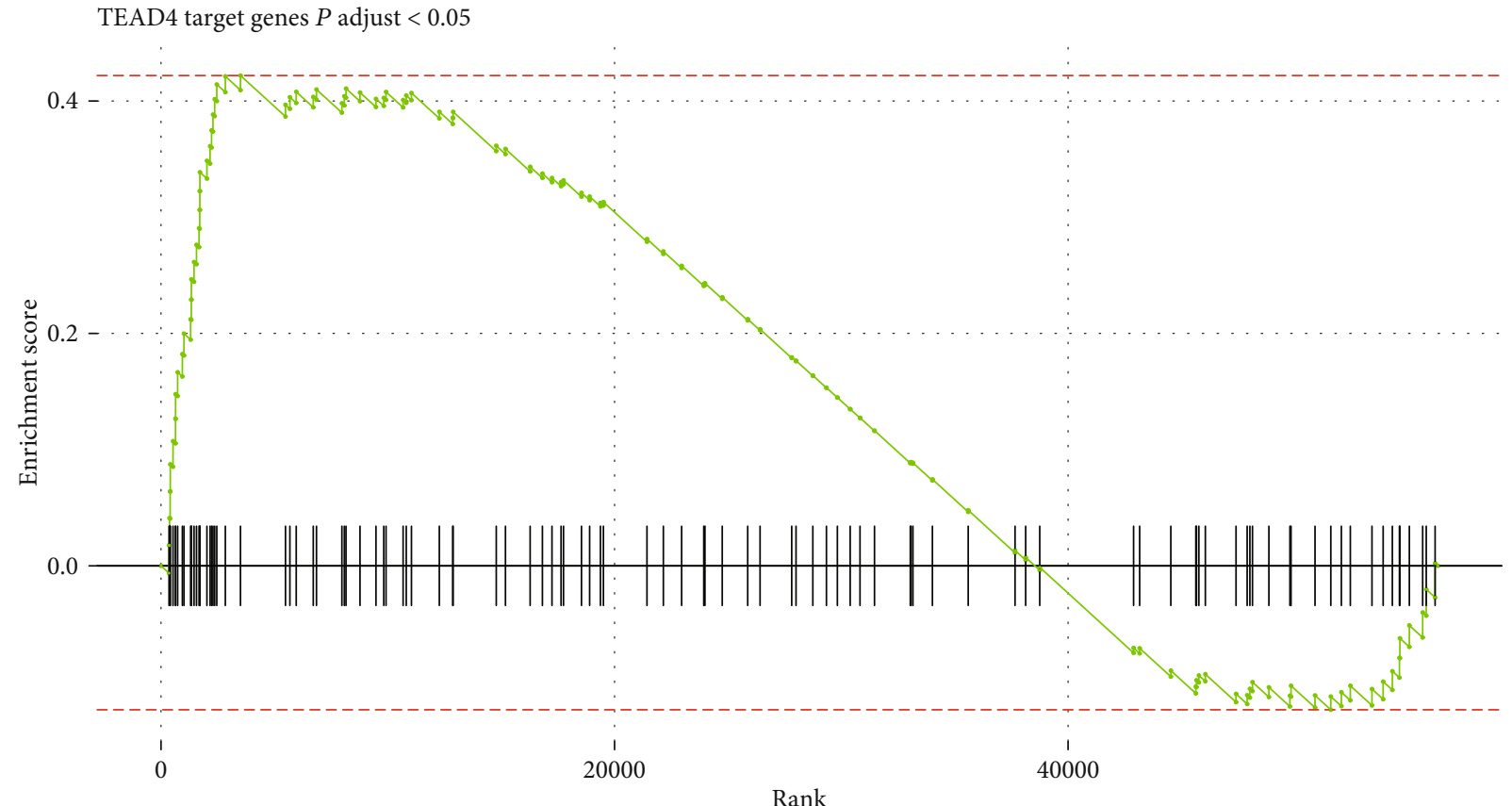

(b)

FIgURE 4: The enrichment degree of the LEF1 and TEAD4 target genes. The enrichment degree of the LEF1 and TEAD4 target genes is displayed in (a) and (b), respectively. The upregulated and downregulated target genes are represented by the black lines on the left and right, respectively.

3.5. The Clinical Significance of the CNA-Related Driver Genes in ESCC. To further explore the clinical significance of identified driver genes, we conducted survival analysis to identify those associated with ESCC overall survival. Among the six driver genes, FGFR3 and EIF4EBP1 were observed to be significantly associated with shorter overall survival in ESCC (Figures 5(a) and 5(b), $P<0.1$, Figure S2). Moreover, ESCC samples with higher expression of the two oncogenes might have a worse prognosis than those with lower expression. These results suggested that FGFR3 and EIF4EBP1 might be unfavorable indicators in ESCC.

\section{Discussion}

Esophageal squamous cell carcinoma (ESCC) is one of the most common malignancies worldwide [17]. ESCC is mostly 


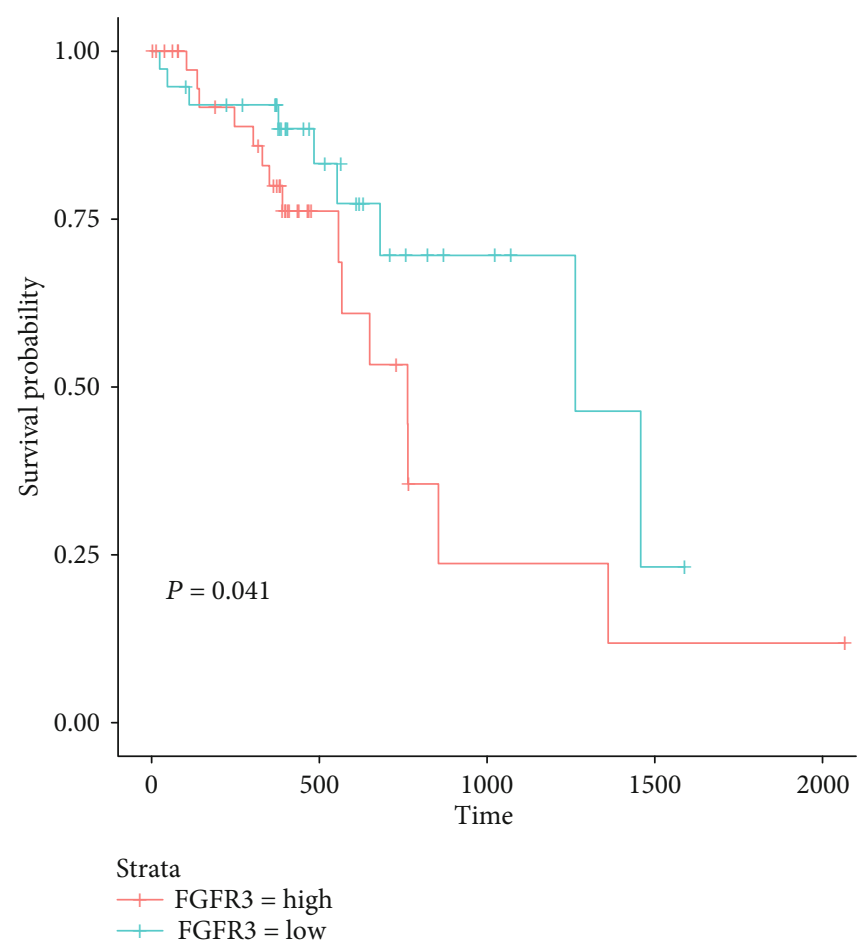

(a)

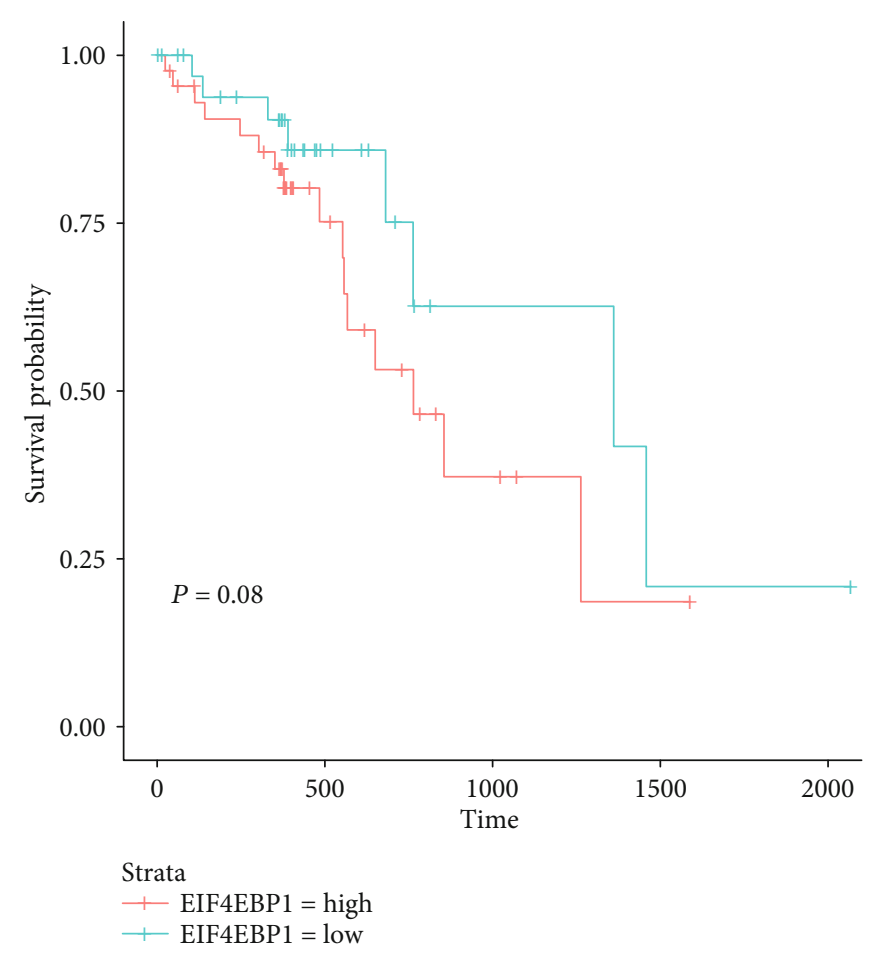

(b)

FIGURE 5: The Kaplan-Meier (KM) curves of the samples with high and low expression of driver genes. The probabilities of the samples with high and low expression of FGFR3 (a) and EIF4EBP1 (b), which are termed as high and low. The statistical significance was tested by the log-rank test.

diagnosed at an advanced stage due to late diagnosis, leading to poor prognoses in ESCC patients [18]. However, the molecular mechanisms of the poor prognosis in ESCC are still unclear. In this study, we aimed to identify and interpret the driver genes triggered by the copy number alterations (CNAs). Specifically, we identified six driver genes including CCND1, TEAD4, EIF4EBP1, EGFR, FGFR3, and FZD6, which were highly upregulated in the samples with gains of the corresponding genes $(P<0.05)$. In the validation dataset, the six candidate driver genes were also upregulated in tumor tissues. These driver genes were involved in the oncogenic pathways including RTK-RAS, WNT, PI3K, Hippo, and cell cycle $[7,19]$. Particularly, EGFR, FGFR3, and FZD6 were receptors of the signaling transduction pathways such as the RTK-RAS and WNT pathways. The CCND1 involved in cell cycle progression; TEAD4, a transcription factor in the Hippo signaling pathway; and EIF4EBP1, the translational regulator in PI3K/Akt/mTOR signaling, have been found to be significantly deregulated in several cancers [2022]. In ESCC, CCND1 was associated with high proliferation [23], and EIF4EBP1 was an adverse prognostic indicator [24, 25]. Furthermore, to verify the consequences of the CNArelated driver genes, we examined the activities of the downstream pathways and transcription factors (TFs). Notably, the genes involved in WNT, NOTCH, and cell cycle were significantly upregulated in the ESCC samples. In addition, the target genes of TFs such as LEF1 and TEAD4 were found to be differentially expressed in ESCC samples. Based on the ORA and GSEA, we found that the upregulated genes were significantly enriched in the components of the oncogenic pathways and the target genes of LEF1 and TEAD4, respectively, suggesting that the WNT, NOTCH, cell cycle, and Hippo signaling pathways were abnormally activated by their corresponding driver genes [26, 27]. These oncogenic signaling pathways have been recurrently reported to lead to uncontrolled proliferation of cancer cells and unfavorable prognostic outcomes in several cancer types [28]. Remarkably, the transcription factor LEF1 has been found to enhance beta-catenin translocation to the nucleus in ESCC [29]. TEAD4 acted as a key regulator of the functional module in ESCC by bioinformatic analysis [30]. The ligands, receptors, and TFs transported to the nucleus in the WNT signaling pathway played key roles in the tumorigenesis and progression of ESCC [31,32]. In addition, we observed that upregulation of FGFR3 was significantly associated with shorter overall survival in ESCC. Enhanced FGFR3 expression promotes high proliferation and causes poor prognosis in ESCC $[33,34]$. Hereby, we speculated that the CNAs in the six driver genes might enhance their expression levels; activate the downstream oncogenic signaling pathways such as WNT, NOTCH, cell cycle, and Hippo via regulating their TFs such as LEF1 and TEAD4, as well as TF target genes; and consequently result in poor prognosis.

Collectively, the driver genes triggered by CNAs not only exhibited critical functionality but also were clinically relevant in ESCC, which greatly improved our understanding of the molecular mechanisms in ESCC. 


\section{Data Availability}

The raw data can be accessed from TCGA.

\section{Conflicts of Interest}

All authors claimed no competing conflicts of interest.

\section{Supplementary Materials}

Figure S1: the expression levels of the six candidate driver genes in ESCC and normal tissues of an independent dataset. The red and grey boxes represent the ESCC and normal tissues, respectively. The mRNA intensities were used as the expression levels. Figure S2: the Kaplan-Meier (KM) curves of the samples with high and low expression of FZD1, FGFR, TEAD4, and CCND1. The red and green curves represent the groups of patients with high and low gene expression. (Supplementary Materials)

\section{References}

[1] F. Bray, J. Ferlay, I. Soerjomataram, R. L. Siegel, L. A. Torre, and A. Jemal, "Global cancer statistics 2018: GLOBOCAN estimates of incidence and mortality worldwide for 36 cancers in 185 countries," CA: A Cancer Journal for Clinicians, vol. 68, no. 6, pp. 394-424, 2018.

[2] Y. Sasaki, M. Tamura, R. Koyama, T. Nakagaki, Y. Adachi, and T. Tokino, "Genomic characterization of esophageal squamous cell carcinoma: insights from next-generation sequencing," World Journal of Gastroenterology, vol. 22, no. 7, pp. 2284-2293, 2016.

[3] Y. Zhang, "Epidemiology of esophageal cancer," World Journal of Gastroenterology, vol. 19, no. 34, pp. 5598-5606, 2013.

[4] S. M. Dawsey, K. J. Lewin, G. Q. Wang et al., "Squamous esophageal histology and subsequent risk of squamous cell carcinoma of the esophagus. A prospective follow-up study from Linxian, China," Cancer, vol. 74, no. 6, pp. 1686-1692, 1994.

[5] F. Tanaka, K. Yamamoto, S. Suzuki et al., "Strong interaction between the effects of alcohol consumption and smoking on oesophageal squamous cell carcinoma among individuals with ADH1B and/or ALDH2 risk alleles," Gut, vol. 59, no. 11, pp. 1457-1464, 2010.

[6] X. C. Li, M. Y. Wang, M. Yang et al., “A mutational signature associated with alcohol consumption and prognostically significantly mutated driver genes in esophageal squamous cell carcinoma," Annals of Oncology, vol. 29, no. 4, pp. 938-944, 2018.

[7] Y. B. Gao, Z. L. Chen, J. G. Li et al., "Genetic landscape of esophageal squamous cell carcinoma," Nature Genetics, vol. 46, no. 10, pp. 1097-1102, 2014.

[8] D. C. Lin, J. J. Hao, Y. Nagata et al., "Genomic and molecular characterization of esophageal squamous cell carcinoma," Nature Genetics, vol. 46, no. 5, pp. 467-473, 2014.

[9] L. Zhang, Y. Zhou, C. Cheng et al., "Genomic analyses reveal mutational signatures and frequently altered genes in esophageal squamous cell carcinoma," American Journal of Human Genetics, vol. 96, no. 4, pp. 597-611, 2015.

[10] D. Hempel, T. Wieland, B. Solfrank et al., "Antitumor activity of larotrectinib in esophageal carcinoma with NTRK gene amplification," The Oncologist, vol. 25, no. 6, pp. e881-e886, 2020.

[11] The Cancer Genome Atlas Research Network, "Integrated genomic characterization of oesophageal carcinoma," Nature, vol. 541, no. 7636, pp. 169-175, 2017.

[12] M. Goldman, B. Craft, M. Hastie et al., "The UCSC Xena platform for public and private cancer genomics data visualization and interpretation," bioRxiv, 2019.

[13] J. Wen, H. Yang, M. Z. Liu et al., "Gene expression analysis of pretreatment biopsies predicts the pathological response of esophageal squamous cell carcinomas to neo-chemoradiotherapy," Annals of Oncology, vol. 25, no. 9, pp. 1769-1774, 2014.

[14] J. Harrow, A. Frankish, J. M. Gonzalez et al., "GENCODE: the reference human genome annotation for the ENCODE Project," Genome Research, vol. 22, no. 9, pp. 1760-1774, 2012.

[15] G. Yu, L. G. Wang, Y. Han, and Q. Y. He, "clusterProfiler: an R package for comparing biological themes among gene clusters," OMICS, vol. 16, no. 5, pp. 284-287, 2012.

[16] C. Gu, X. Shi, Z. Huang et al., "A comprehensive study of construction and analysis of competitive endogenous RNA networks in lung adenocarcinoma," Biochimica et Biophysica Acta (BBA) - Proteins and Proteomics, vol. 1868, no. 8, article 140444, 2020.

[17] A. Pennathur, M. K. Gibson, B. A. Jobe, and J. D. Luketich, "Oesophageal carcinoma," Lancet, vol. 381, no. 9864, pp. 400-412, 2013.

[18] E. C. Smyth, J. Lagergren, R. C. Fitzgerald et al., "Oesophageal cancer," Nature Reviews Disease Primers, vol. 3, no. 1, article 17048, 2017.

[19] M. Secrier, X. Li, N. de Silva et al., "Mutational signatures in esophageal adenocarcinoma define etiologically distinct subgroups with therapeutic relevance," Nature Genetics, vol. 48, no. 10, pp. 1131-1141, 2016.

[20] E. A. Musgrove, C. E. Caldon, J. Barraclough, A. Stone, and R. L. Sutherland, "Cyclin D as a therapeutic target in cancer," Nature Reviews Cancer, vol. 11, no. 8, pp. 558-572, 2011.

[21] Y. Zhou, T. Huang, A. Cheng, J. Yu, W. Kang, and K. To, “The TEAD family and its oncogenic role in promoting tumorigenesis," International Journal of Molecular Sciences, vol. 17, no. 1, p. 138, 2016.

[22] A. C. Rutkovsky, E. S. Yeh, S. T. Guest et al., "Eukaryotic initiation factor 4E-binding protein as an oncogene in breast cancer," BMC Cancer, vol. 19, no. 1, p. 491, 2019.

[23] H. Chang, J. Song, J. Wu, and Y. Zhang, "E2F transcription factor 8 promotes cell proliferation via CCND1/p 21 in esophageal squamous cell carcinoma," Oncotargets and Therapy, vol. Volume 11, pp. 8165-8173, 2018.

[24] Y. K. Chao, W. Y. Chuang, C. J. Yeh et al., "High phosphorylated $4 \mathrm{E}$-binding protein 1 expression after chemoradiotherapy is a predictor for locoregional recurrence and worse survival in esophageal squamous cell carcinoma patients," Journal of Surgical Oncology, vol. 105, no. 3, pp. 288-292, 2012.

[25] C. J. Yeh, W. Y. Chuang, Y. K. Chao et al., "High expression of phosphorylated $4 \mathrm{E}$-binding protein 1 is an adverse prognostic factor in esophageal squamous cell carcinoma," Virchows Archiv, vol. 458, no. 2, pp. 171-178, 2011.

[26] Y. Zhao, C. Li, L. Huang et al., "Prognostic value of association of OCT4 with LEF1 expression in esophageal squamous cell carcinoma and their impact on epithelial-mesenchymal transition, invasion, and migration," Cancer Medicine, vol. 7, no. 8, pp. 3977-3987, 2018. 
[27] J. Li, Z. Li, Y. Wu et al., "The Hippo effector TAZ promotes cancer stemness by transcriptional activation of SOX2 in head neck squamous cell carcinoma," Cell Death \& Disease, vol. 10, no. 8, p. 603, 2019.

[28] F. Sanchez-Vega, M. Mina, J. Armenia et al., "Oncogenic signaling pathways in the Cancer Genome Atlas," Cell, vol. 173, no. 2, pp. 321-337.e10, 2018.

[29] S. Tanaka, T. Akiyoshi, M. Mori, J. R. Wands, and K. Sugimachi, "A novel frizzled gene identified in human esophageal carcinoma mediates APC/beta-catenin signals," Proceedings of the National Academy of Sciences of the United States of America, vol. 95, no. 17, pp. 10164-10169, 1998.

[30] X. Wang, G. Li, Q. Luo, and C. Gan, "Identification of crucial genes associated with esophageal squamous cell carcinoma by gene expression profile analysis," Oncology Letters, vol. 15, no. 6, pp. 8983-8990, 2018.

[31] J. Zhang, J. L. Wang, C. Y. Zhang, Y. F. Ma, R. Zhao, and Y. Y. Wang, "The prognostic role of FZD6 in esophageal squamous cell carcinoma patients," Clinical \& Translational Oncology, vol. 22, no. 7, pp. 1172-1179, 2020.

[32] Y. Z. Deng, P. P. Chen, Y. Wang et al., "Connective tissue growth factor is overexpressed in esophageal squamous cell carcinoma and promotes tumorigenicity through betacatenin-T-cell factor/Lef signaling," The Journal of Biological Chemistry, vol. 282, no. 50, pp. 36571-36581, 2007.

[33] N. Ueno, A. Shimizu, M. Kanai et al., "Enhanced expression of fibroblast growth factor receptor 3 IIIc promotes human esophageal carcinoma cell proliferation," The Journal of Histochemistry and Cytochemistry, vol. 64, no. 1, pp. 7-17, 2015.

[34] H. S. Kim, S. E. Lee, Y. S. Bae et al., "Fibroblast growth factor receptor 1 gene amplification is associated with poor survival in patients with resected esophageal squamous cell carcinoma," Oncotarget, vol. 6, no. 4, pp. 2562-2572, 2015. 